\title{
Detection and phylogenetic profiling of nodavirus associated with white tail disease in Malaysian Macrobrachium rosenbergii de Man.
}

\begin{abstract}
White tail disease (WTD) is a serious viral disease in the hatcheries and nursery ponds of Macrobrachium rosenbergii in many parts of the world. A new disease similar to WTD was observed in larvae and post larvae of M.rosenbergii cultured in Malaysia. In the present study,RT-PCR assay was used to detect the causative agents of WTD, M.rosenbergii nodavirus (MrNV) and extra small virus (XSV) using specific primers for MrNV RNA2 and XSV. The results showed the presence of MrNV in the samples with or without signs of WTD. However, XSV was only detected in some of the MrNV-positive samples.Phylogenetic analysis showed that the RNA2 of our Malaysian isolates were significantly different from the other isolates. Histopathological studies revealed myofiber degeneration of the tail muscles and liquefactive myopathy in the infected prawns. This was the first report on the occurrence of MrNV in the Malaysian freshwater prawn.
\end{abstract}

Keyword: Macrobrachium rosenbergii; Nodavirus; White tail disease; RT-PCR; Histopathology. 